\title{
EDITORIAL More on the clip versus coil controversy
}

\author{
Eric C. Peterson, MD, and Roberto C. Heros, MD \\ Department of Neurological Surgery, University of Miami Miller School of Medicine, Miami, Florida
}

$\mathrm{T}$ HE issue of whether to clip or coil a ruptured intracranial aneurysm continues to be debated. The preponderance of the evidence in the literature indicates that under equal circumstances (and circumstances are rarely equal for individual patients) patients treated with coils tend to have a better outcome at mid-term follow-up (1 year). In this issue of the Journal, Ayling and colleagues address the important considerations of whether there is a difference in the immediate postoperative outcome, what are the factors that lead to postoperative deterioration with each therapeutic maneuver, and whether postoperative deterioration is related to long-term outcome. ${ }^{1}$ They also make a good attempt at comparing clipping and coiling in these respects. To this end they conducted a very thoughtful analysis of the prospectively collected data set from the Clazosentan to Overcome Neurological iSChemia and Infarction Occurring after Subarachnoid hemorrhage (CONSCIOUS-1) study, a randomized study of the effect of clazosentan on delayed ischemia after subarachnoid hemorrhage (SAH). It is important to be reminded that in this study, which included both patients treated with coils and those who underwent clipping, the patients were randomized to receive clazosentan or placebo; they were not randomized by modality of treatment. To approximate randomization as much as one can by retrospectively looking at a data set like this, the authors used the very elegant statistical maneuver of propensity-score matching. Clearly, this or any other statistical analysis used post hoc does not substitute perfectly for prospective randomization.

The findings of the authors can be summarized as follows: 1) patients who underwent clipping had an increased incidence of postoperative decline as compared to those treated with coils; 2) of the many factors analyzed, the ones found to be independently significant in determining immediate postoperative deterioration were intraoperative hypertension or induced hypotension in patients treated with clipping and thromboembolic events in those who underwent coiling; and 3) immediate postoperative deterioration in both cohorts of patients strongly correlated with outcome at 3 months. It should be said that none of these findings are surprising; however, they have not been studied in this careful manner before.

Some comments are in order. First and foremost it should be kept in mind, as mentioned above, that patients in the CONSCIOUS-1 study were not randomized by modality of treatment but rather by whether or not they were administered clazosentan. The analysis used by the authors to compare clipping and coiling, good as it is, is no more than what could be described as "pseudorandomization," and the results should be interpreted in this context. The authors use the Glasgow Coma Scale (GCS) score, which was carefully recorded before and immediately after treatment and for several days thereafter to measure postoperative deterioration. Of course, this is what was available to them from the data of the CONSCIOUS-1 study and they could do no better. However, we need to remind ourselves that the GCS was designed for head-injured patients and is not a sensitive enough scale to judge postoperative deterioration. For example, the patient could have a major focal deficit, such as complete hemiplegia, and still be perfectly awake and oriented and with normal eye movements, which would give him a perfect score of 15 in the GCS. It is possible that significant focal deficits occurred both in the patients who underwent clipping and those treated with coils that would not have been reflected in a change in the GCS score. Along the same lines, the dichotomized extended Glasgow Outcome Scale is likely to oversimplify differences in outcome; there is a major difference between being perfect at 3 months and being independent with "moderate disability" (both would be classified as a good outcome); likewise there is a significant difference between being partially dependent and being in a vegetative state (both would be classified as a "poor outcome"). 
We are surprised that induced hypotension was still used with some frequency at the time that this study was conducted. Certainly such a practice has been discredited for many years, and hopefully the results of this study will further discourage the use of intentional intraoperative hypotension. The correlation between intraoperative hypertension and postoperative decline, which was not as robust as the correlation with hypotension, is interesting and perhaps less predictable. It is doubtful that some degree of hypertension intraoperatively was directly responsible for neurological deterioration, and one has to wonder whether that was not an epiphenomenon of other intraoperative events such as aneurysmal rupture or brain swelling. It is somewhat surprising that other intraoperative events such as aneurysmal rupture and prolonged temporary occlusion were not statistically correlated to postoperative deterioration, although, as the authors explain, this may have been due to the small number of such events.

Has this study resolved the issue of clip versus coil? Certainly not; however, we do believe that this well-performed analysis significantly reinforces the emerging consensus that under equal circumstances intraoperative deterioration occurs more frequently with clipping than with coiling, and that such immediate postoperative deterioration is strongly correlated with outcome at 3 months. What this study does not do is to relieve the neurosurgeon from the need to analyze very carefully in each patient the factors and circumstances that make that patient different from any other. For example, what is the patient's age? Clearly elderly patients tend to tolerate open surgery less well, which would favor coiling, whereas younger patients have longer to live, which would make the long-term permanency of clipping more attractive. Certain aneurysms are more favorable for coiling, and others can be treated more satisfactorily at the present time by open craniotomy. The treating team may be more experienced and adept at one form of treatment or the other. The patient with a poor neurological grade, indicative of a swollen brain, may be best treated endovascularly, where the brain does not need to be disturbed. An aneurysm at the middle cerebral artery bifurcation can be exposed much more safely at craniotomy than an aneurysm at the basilar bifurcation, where almost always an endovascular technique is nowadays preferable. To this we need to add the importance of evolution in the technology, which, for example, makes it possible today to treat with low-porosity stents a complex large or giant aneurysm that until recently had to be treated by open craniotomy, with or without a bypass graft and complex clipping techniques.

We heartily congratulate Dr. Macdonald, Mr. Ayling, and their colleagues for an excellent attempt to add more clarity to this topic.

http://thejns.org/doi/abs/10.3171/2014.12.JNS142536

\section{Reference}

1. Ayling OGS, Ibrahim GM, Drake B, Torner JC, Macdonald RL: Operative complications and differences in outcome after clipping and coiling of ruptured intracranial aneurysms. J Neurosurg [epub ahead of print June 5, 2015. DOI: 10.3171/2014.11.JNS141607]

\section{Response}

Oliver G. S. Ayling, MSc, George M. Ibrahim, MD, PhD, and R. Loch Macdonald, MD, PhD

Division of Neurosurgery, St. Michael's Hospital, Labatt Family Centre of Excellence in Brain Injury and Trauma Research, Keenan Research Centre for Biomedical Science, Li Ka Shing Knowledge Institute, St. Michael's Hospital and Department of Surgery, University of Toronto, Toronto, Ontario, Canada

We thank Drs. Peterson and Heros for their thoughtful commentary, informed by rigorous knowledge of the literature and years of neurosurgical expertise. Endovascular coiling is the modality of choice in circumstances where equipoise is present between the 2 procedures. As Drs. Peterson and Heros point out, to conclude that coiling is universally superior to microsurgical clipping is inaccurate and provides a disservice to patients affected by aneurysmal SAH. The broadening of indications for coiling that is occurring is not supported by the randomized controlled clinical trials data that led to the initial approval for coils. Rather, as Peterson and Heros note, it is based on patient selection, and on understanding and mitigating the negative sequelae associated with both treatment modalities. But this is basically individual doctor opinion that is not a reliable basis for treatment recommendations.

In the investigation reported in the current issue of the Journal, we performed a pseudorandomized post hoc analysis of the CONSCIOUS-1 trial to study differences in postoperative neurological deterioration among patients undergoing clipping and coiling, the factors associated with this decline, and the contributions of this decline to neurological outcomes at 3 months. A study was recently published showing perioperative neurological deterioration in patients who underwent clipping, ${ }^{2}$ but ours is the first to directly compare this deterioration in prospective cohorts treated with one or the other of these modalities and to study its effect on overall outcome. Our findings underline the importance of the perioperative period when considering delayed outcomes and comparing the 2 procedures. These findings are particularly relevant, given that numerous factors may modify outcome after clipping and coiling, including the incidence of angiographic vasospasm and delayed cerebral ischemia. ${ }^{1}$

The comments about the limitations of the GCS and outcome scores are right on the mark. Development of SAH-specific neurological assessment and outcome scales is underway as part of a cooperative group that we are organizing. We agree with Drs. Peterson and Heros that the conclusions that can be drawn from our data set are limited by the post hoc nature of the study, the variables available for analysis, and the original intent of the CONSCIOUS-1 trial, which was not to compare clipping and coiling. With these limitations in mind, we showed that periprocedural neurological deterioration is an important mitigating factor that may explain differences in outcomes between the 2 treatment modalities. Our study also highlights practices that could be avoided (keeping in mind the nonrandomized data), such as induced hypotension during clipping, and areas where further research to mitigate complications are necessary, such as thromboembolic events during coiling. 
We certainly agree that intraoperative hypertension, which was also associated with poor outcomes in patients undergoing clipping, may be related to other events that were not directly measured, including early brain injury, ${ }^{3}$ intraoperative aneurysm rupture, or prolonged temporary artery occlusion. We suggest that this post hoc analysis is not definitive in any way but that it is hypothesis generating and can support the conduct of prospective randomized studies aimed at improving the outcome of patients with SAH. Although this study does not necessarily make the decision to clip or coil any easier, we were able to shed some light on the importance of the perioperative period contributing to overall outcome by directly comparing patients who underwent surgical clipping with those treated with endovascular coiling; patients who were recruited within the same study cohort. As new endovascular technology continues to emerge, making it possible to treat complex or large aneurysms that were traditionally clipped, it will be important for future studies and prospective databases to examine not only the long-term outcomes but also perioperative variables that may assist neurosurgeons in minimizing perioperative neurological decline.

\section{References}

1. Ibrahim GM, Vachhrajani S, Ilodigwe D, Kassell NF, Mayer SA, Ruefenacht D, et al: Method of aneurysm treatment does not affect clot clearance after aneurysmal subarachnoid hemorrhage. J Neurosurg 70:102-109, 2012

2. Mahaney KB, Todd MM, Bayman EO, Torner JC: Acute postoperative neurological deterioration associated with surgery for ruptured intracranial aneurysm: incidence, predictors, and outcomes. J Neurosurg 116:1267-1278, 2012

3. Sehba FA, Hou J, Pluta RM, Zhang JH: The importance of early brain injury after subarachnoid hemorrhage. Prog Neurobiol 97:14-37, 2012 